\title{
Biospeologica sovietica.XLIX. Le premier représentant anophtalme des Trechini (Coléoptères Carabiques) de la Ciscaucasie
}

\author{
par
}

\section{S.I.LJOVUSCHKIN*}

En 1970, l'auteur du présent article et l'étudiant en Zoologie M.A.Snetkov ont étudié une série de grottes dans la Ciscaucasie occidentale.

Dans une des grottes nous avons récolté deux exemplaires de Coléoptères appartenant au genre nouveau de Trechini. Cela représente d'autant plus d'intérêt qu'avant ce temps “aucune espèce endémique de Trechini n'est connue des montagnes de la Ciscaucasie" (Jeannel, 1960, p.211).

Le genre nouveau est nommé en mémoire du Prof.J.A.Birstein, biospéléologiste soviétique célèbre.

\section{DIAGNOSE DE BIRSTEINIOTRECHUS LJOVUSCHKIN N.GEN.}

Genre de la sérié phylétique de Neotrechus, voisin de Troglocimmerites Ljov., mais qui diffère grandement de celui-ci par ses caractères labiaux.

Taille petite. Téguments dépigmentés.

Tête arrondie, les sillons frontaux incomplets. Yeux totalement oblitérés, leur place n'est marquée que par un trait préoculaire. Antennes modérément épaisses, sans atteindre le milieu des ély tres.

Labre échancré, armé de 6 soies, dont les latérales sont plus longues que les médianes. Mandibules courtes, à rétinacles saillants, celle de droite est trituberculée. Palpes maxillaires courts et épais, l'avant-dernier article très renflé, le dernier plus mince et court que l'avant-dernier. Labium libre, la dent médiane simple est très saillante, six soies labiales, dont les latérales relativement courtes (fig.2.1) Languette à bord libre transversal munie de deux grandes soies médianes, de quatre soies marginales plus courtes et de deux très courtes disposées près des soies médianes (fig. 2.2).

Pronotum cordiforme plus large que long, très rétréci à la base: angles postérieurs saillants et pointus (fig.1.2). Elytres ovales, allongés et pubescents: épaules effacées, leur bord marginal crénelé et cilié (fig.1.3).

Les pattes modérément longues et grêles. Tibias sans trace de sillon longitudinal externe. Les mâles possèdent un seul article dilaté aux tarses antérieurs (fig.2.3).

Chétotaxie.- Deux soies sous-orbitaires. Soies prothoraciques normales, l'anté-

* Faculté de Biologie, Université de Moscou, Moscou 117234, URSS. 
rieure disposée au quart antérieur du côté, la postérieure avant l'angle postérieur. Deux soies discales sur la $3 \mathrm{e}$ interstrie des élytres. Triangle apical complet. Série ombiliquée plus ou moins agrégée dans la gouttière marginale, mais quelques fouets sont écartés de la gouttière marginale (fig.1.3).

Organe copulateur grêle et très arqué. Le bulbe basal porte un aileron sagittal. Styles larges et relativement courts, avec 7 ou 8 soies apicales. Sac interne comporte une pièce copulatrice (fig.2.4).

Type: Birsteiniotrechus ciscaucasiens Ljovuschkin sp.n.

\section{Affinités}

Sans doute le genre Birsteiniotrechus fait partie de la série phylétique de Neotrechus, caractérisé par les yeux totalement disparus, la soie prothoracique postérieure avant l'angle postérieur et un seul article dilaté aux tarses antérieurs mâles

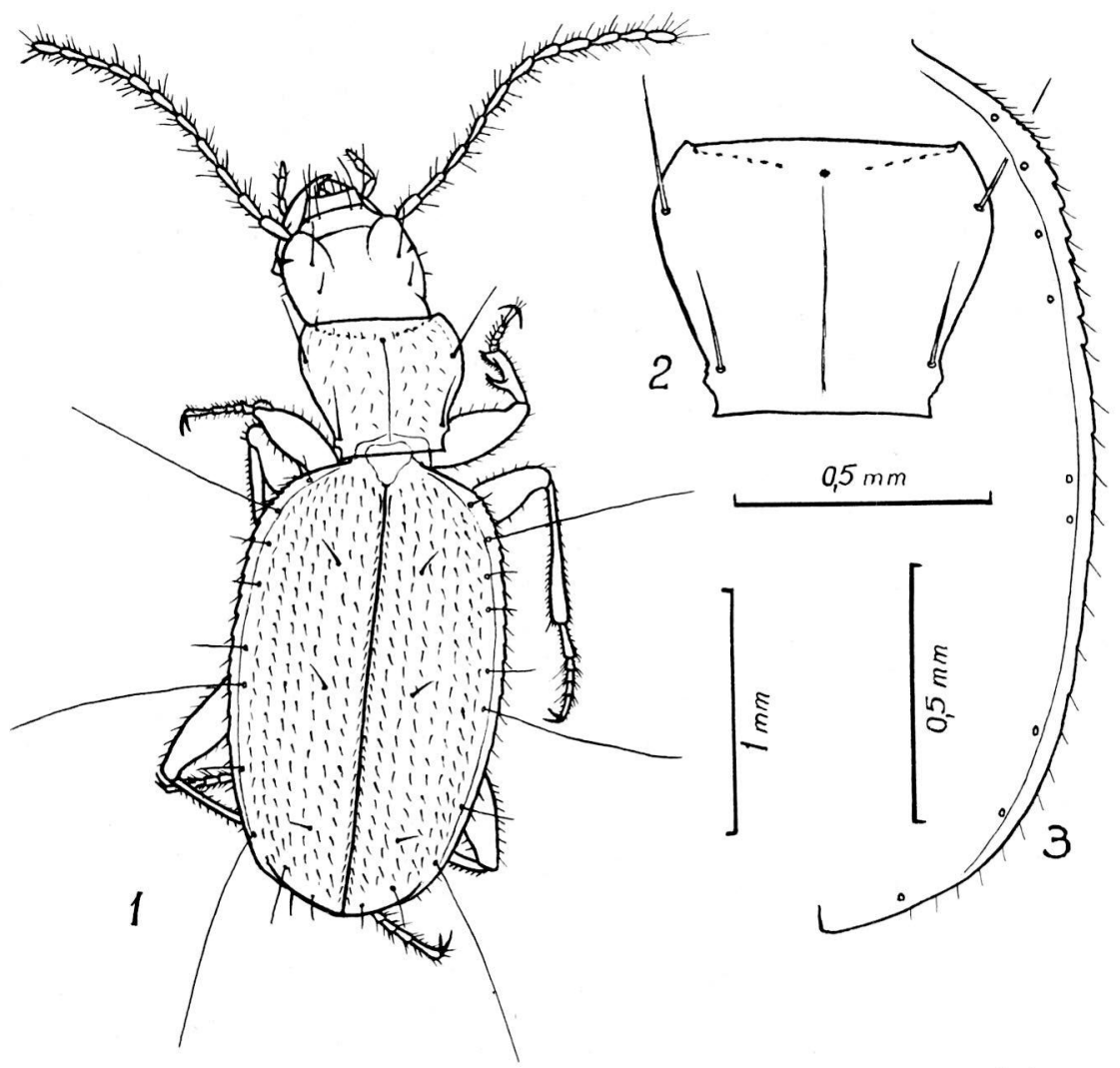

Fig.1. Birsteiniotrechus ciscaucasiens gen. et sp.n., ơ Holotype: 1 -aspect général, 2 pronotum, $3-$ le bord des ély tres 
(Jeannel, 1928). Actuellement cette série phylétique comprend 8 genres: Neotrechus J.Müll (Yougoslavie et Albanie du Nord), Orotrechus J.Müll (Italie du Nord et Slovénie), Anillidius Jeann. (partie européenne de la Turquie, Bithynie et Taurus de Pisidie), Kosswigia Jeann. (Taurus de Pisidie), Cimmerites Jeann. (Transcaucasie occidentale), Jeannelius Kurn. (Transcaucasie occidentale), Nannotrechus Winkler (Abkhazie), Troglocimmerites Ljov. (Géorgie: district de Ambrolaouri) (Jeannel, 1928, 1947, 1960; Kurnakov, 1959; Ljovuschkin, 1970).

Les genres du Caucase et de la région pontique, y compris le genre nouveau, sont parents. A l'exception de Kosswigia, ils différent des genres Neotrechus et Orotrechus par la série ombliquée plus ou moins agrégée. Tous les genres occidentaux ont les tibias antérieurs non sillonés (Orotrechus a les tibias antérieurs sillonés). La différence entre les genres de la série phylétique de Neotrechus connus du Caucase, de l'Asie Mineure et de la partie européenne de la Turquie est la suivante:

\begin{tabular}{|c|c|c|c|c|c|c|}
\hline $\begin{array}{l}\text { Anilli- } \\
\text { dius }\end{array}$ & $\begin{array}{l}\text { Cimme- } \\
\text { rites }\end{array}$ & $\begin{array}{l}\text { Jeanne- } \\
\text { lius }\end{array}$ & $\begin{array}{l}\text { Birs- } \\
\text { teinio- } \\
\text { trechus }\end{array}$ & $\begin{array}{l}\text { Troglo- } \\
\text { cimme- } \\
\text { rites }\end{array}$ & $\begin{array}{l}\text { Nanno- } \\
\text { trechus }\end{array}$ & $\begin{array}{l}\text { Kosswi- } \\
\text { gia }\end{array}$ \\
\hline libre & libre & libre & libre & soudé & soudé & soudé \\
\hline bifide & bifide & bifide & simple & bifide & simple & simple \\
\hline comple & $\begin{array}{l}\text { scom- } \\
\text { plets }\end{array}$ & $\begin{array}{l}\text { incom- } \\
\text { plets }\end{array}$ & $\begin{array}{l}\text { incom- } \\
\text { plets }\end{array}$ & $\begin{array}{l}\text { incom- } \\
\text { plets }\end{array}$ & $\begin{array}{l}\text { com- } \\
\text { plets }\end{array}$ & $\begin{array}{l}\text { com- } \\
\text { plets }\end{array}$ \\
\hline agrégée & $\begin{array}{l}\text { agré- } \\
\text { gée }\end{array}$ & $\begin{array}{l}\text { agré- } \\
\text { gée }\end{array}$ & $\begin{array}{l}\text { agré- } \\
\text { gée }\end{array}$ & $\begin{array}{l}\text { agré- } \\
\text { gée }\end{array}$ & $\begin{array}{l}\text { agré- } \\
\text { gée }\end{array}$ & $\begin{array}{l}\text { non } \\
\text { agrégée }\end{array}$ \\
\hline
\end{tabular}

1. Labium libre libre libre libre soudé soudé soudé

2. Dent labiale

3. Sillons frontaux

4. Série ombiliquée absente présente absente présente présente absente absente ce de l'élytre

6. Bord marginal de l'élytre lisse crénelé lisse crénelé peu crénelé lisse lisse

7. 1er article du tarse anté dilaté simple simple dilaté dilaté dila- dilarieur mâle

8. Piêce copulatrice présente absente présente présente présente présente présente 


\section{DIAGNOSE DE BIRSTEINIOTRECHUS CISCAUCASIENS LJOVUSCHKIN N.SP.}

Cette espèce a été récoltée le 4 août 1970. Les deux exemplaires (200*) ont été trouvés dans la grotte "Ammonitovaja peštera" près du hameau Chedok dans les environs du bourg Psebaj (district Laba, région de Krasnodar, dans le bassin du Kouban). Holotype et paratype au Museum Zoologique de Moscou.

\section{Description}

Longueur de $3 \mathrm{~mm}$ (paratype) à 3,1 mm (holotype). Testacé, d'un roux pâle. Les temps, le pronotum et surtout les élytres couverts de poils hérissés. Épais et allongés, les téguments alutacés.

Tête petite, aussi longue que le pronotum. Les sillons frontaux incomplets et
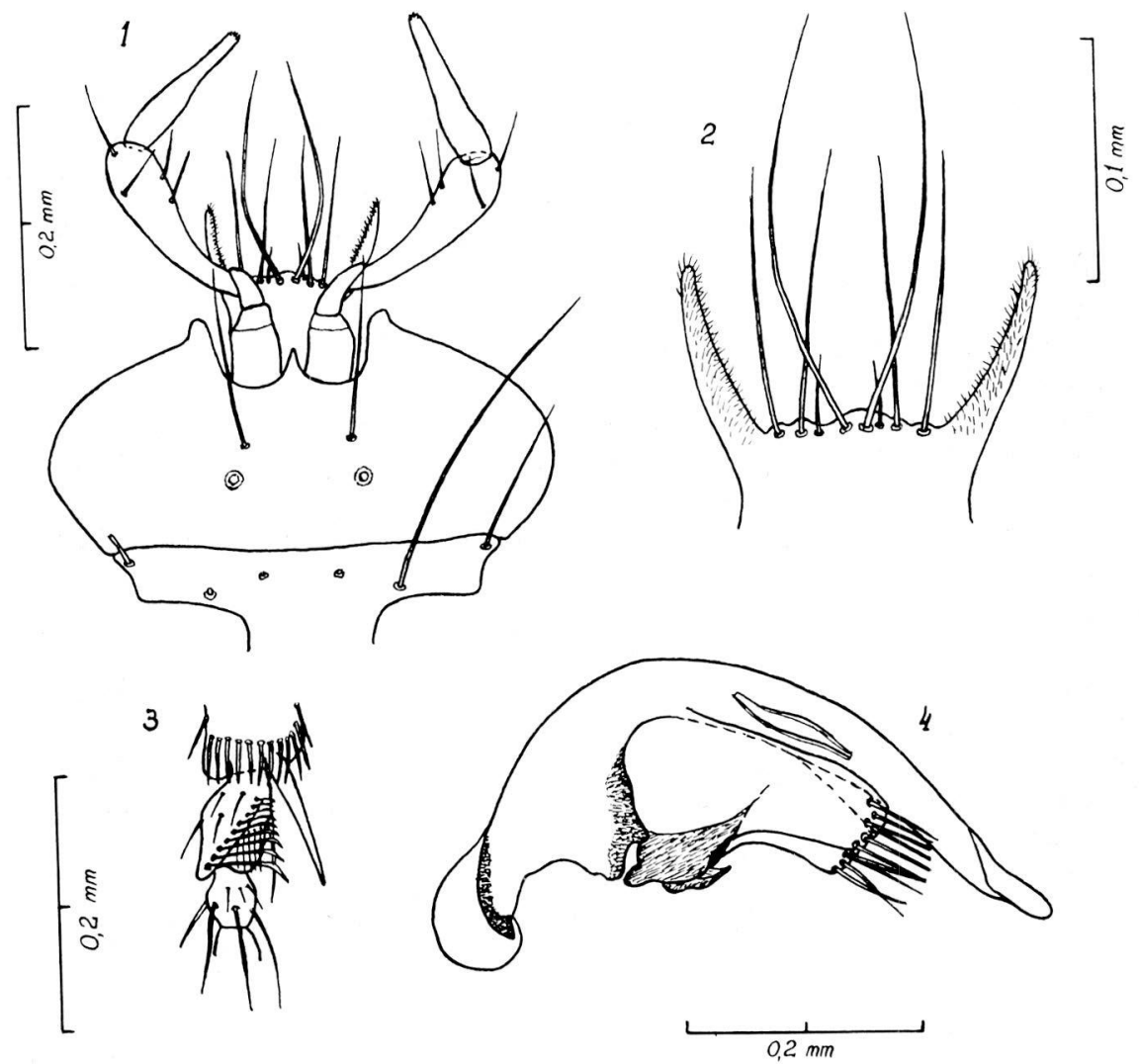

Fig. 2. Birsteiniotrechus ciscaucasiens gen. et sp.n., o Paratype: 1 - labium, 2 - languette, 3.ler article du tarse, 4-organe copulateur. 
arqués. Pas de traces d'yeux. Articles apicaux des antennes ovales, le dernier article plus long que l'avant-dernier. Pièces buccales robustes et courtes Cou très épais.

Pronotum cordiforme; les côtés sont arrondis, avec les saillants dans le cinquième basal, les angles antérieurs saillants mais émoussés; les angles postérieurs aigus et saillants; la base rectiligne; disque convexe, la ligne médiane fine; les fossettes basales superficielles; la gouttière marginale régulière.

Elytres oblongs, à épaules très arrondies; disque convexe; la gouttière marginale étroite. Stries superficielles et effacées. Aptère.

Les tibias antérieurs non sillonnés et pubescents sur leurs faces antérieures. Tarses antérieurs mâles avec un seul (le premier) article dilaté, le deuxième petit et arrondi, ressemble au troisième. Tarses postèrieurs grêles.

Chétotaxie. - La première soie discale est disposée au quart antérieur de la $3 \mathrm{e}$ interstrie, postérieure au milieu de la $3 \mathrm{e}$ interstrie. Triangle apical allongé; la soie apicale antérieure éloignée de l'apex dans le 3e strie; la soie externe en face de la carène apicale. Les fouets du groupe huméral de la série ombiliquée équidistants. Le groupe moyen au milieu de l'ély tre.

Edéage avec le bulbe basal renflé et coudé. Styles très larges. Pièce copulatrice étroite, en gouttière, avec les rétrécissements proximal et distal.

\section{REMARQUES BIOGÉOGRAPHIQUES SUR LES TRECHINI CAVERNICOLES DU CAUCASE}

Il y a au Caucase quatre séries phylétiques de Trechini (excepté les formes insertae sedis) qui peuplent. les grottes: Trechus, Aphaenops, Duvalinus et Neotrechus.

La série phylétique des Trechus est représentée par une seule espèce Trechus heniochicus Ljov. connue de la grotte "Vorontzovskaja peštera" près de Sotchi (Ljovuschkin, 1970).

Meganophthalmius mirabilis Kurn. est la seule espèce du Caucase appartenant à la série phylétique des Aphaenops (Kurnakov, 1959; Jeannal, 1966).

L'espèce cavernicole (Duvalius sokolovi Ljov.) et trois espèces endogées (D.antoniae Reit., D.kurnakovi Jeann., et D.stepanavensis Khnz.) se rapportent à la série phylétique des Duvalius (Jeannel, 1960; Jablokoff-Khnozorian, 1963; Ljovuschkin, 1963).

La série phylétique Neotrechus est représentée, au Caucase, plus abondamment que les autres ( 5 genres et 11 espèces), elle inclut les formes hautement spécialisée (celles de l'endogé et des grottes) et parmie lesquelles cette série est la plus répandue (fig. 3 ). Il est à noter qu'en Crimée, dans une autre région méditerranéenne du pays, cette série n'est pas repérée.

Selon l'avis de R.Jeannel (1928) partagé par A.Vandel (1964) les cavernicoles de cette série avaient des ancêtres habitant l'endogé. L'anophtalmie, l'aptérisme et la dépigmentation se sont aussi formées en milieu endogé. Ces caractères sont communs aux espèce cavernicoles et endogées. Les particularités morphologiques qui distinguent des représentants de troglobies de la série phylétique Neotrechus ne sont pas nombreuses (taille plus grande, la série ombiliquée n'est pas agrégée dans la 


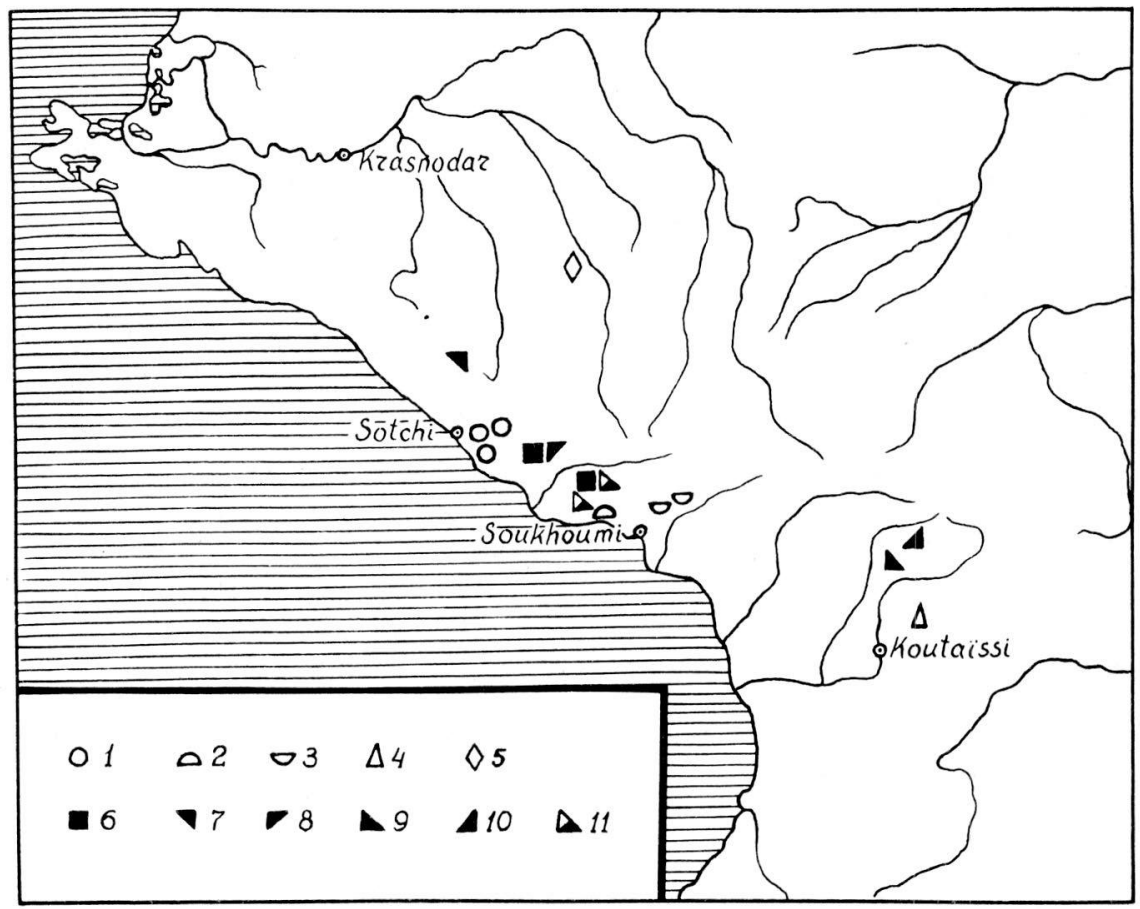

Fig.3. La distribution des representants de serie phyletique de Néotrechus au Caucase occidental (signes noirs - les formes endogées, signes blancs-formes cavernicoles, noirs et blanc - formes trouvées en endogé et dans les grottes): 1- Jeannelius birsteini; 2 - J. gloriosus; 3 - J. magnificus; 4 - Troglocimmerites djanaschvili; 5 - Birsteiniotrechus ciscaucasicus; 6 - Nannotrechus hoppi; 7 - Cimmerites circassicus; 8 - C.serrulatus; 9 C.svaneticus; 10 - C.naceralae; 11 - C.kurnakovi.

gouttière marginale) et ne sont pas propres à tous les troglobiontes de cette série. Notamment Birsteiniotrechus qui ne possède pas les particularités susmentionnées est, problablement, un troglobie habitant à la limite de l'aire de dispersion de la série.

Les espèces des Jeannelius et Troglocimmerites se rapportent aussi aux troglobiontes. Les espèces des Nannotrechus et Cimmerites sont des formes typiques endogées. Parmi ces formes seul C.kurnakovi Jeann. récolté dans les grottes peut être considéré comme troglophile.

Le problème de l'origine des Trechini du Caucase est examiné par R.Jeannel. D'après lui, tous les cavernicoles et les formes spécialisées du milieu endogé représentent "les lignées anciennes, rester de la faune nummulitigne de la Mésogéide" (Jeannel, 1960, p.212).

Les deux groupes du genre Trechus, T.gravidus et T.lederi ont la même origine. Après la parution de l'ouvrage de R.Jeannel, dans les grottes du Caucase ont été 
trouvées 5 nouvelles espèces Trechini. Ce sont les représentants du groupe T.gravidus (T.heniochicus) et de la série phylétique Neotrechus (Jeannelius birsteini Ljov., J.gloriosus l_jov., Troglocimmerites djanaschvili Ljov et Birsteiniotrechus ciscaucasiens; Ljovuschkin, 1963, 1965, 1970). Les trouvailles mentionnées ont augmenté de deux fois la diversité de genres et d'espèces des Trechini des cavernes du Caucase mais elles n'ont pas modifié l'idée du caractère des Trechini, de leurs liens zoogéographiques et de leur origine.

\section{RESUME}

Une exploration récente de grottes des Ciscaucasie occidentale a permis de récolter notamment un genre nouveau de Trechini (Coleoptera, Caraboidea) dont on trouvera ici la description, il est nommé Birsteiniotrechus ciscaucasiens n.gen., n..sp., en mémoire du Prof. J.A.Birstein, biospéléologiste soviétique célèbre, récemment disparu (cf. I.J.S. vol.4, part.2), fondateur et promoteur de la série de travaux intitulée "Biospeologica sovietica".

L'intérêt du Birsteiniotrechus réside dans le fait que jusqu'alors aucune espèce troglobie et endémique de Trechini n'était connue des montagnes de Ciscaucasie, c'est un genre de la série phylétique des Neotrechus, voisin des Troglocimmerites mais en différant grandement par ses caractères labiaux.

\section{SUMMARY}

During a recent exploration of caves in western Ciscaucasia there was collected, among others, a new species representing a new genus of Trechini (Coleoptera, Caraboidea), the description of which is given here; it has been named Birsteiniotrechus ciscaucasiens n.gen., n.sp., in memory of Prof. J.A.Birstein, famous Soviet biospeleologist, the founder and promotor of the "Biospeologica sovietica" series who passed away recently (cf. I.J.S., 4, part.2).

The interest of Birsteiniotrechus lies in the fact that, until now no troglobite or endemic species of Trechini was known from the Ciscaucasian mountains; this genus belongs to the phyletic series of Neotrechus and is nearly allied to Troglocimmerites, but differs from it by its labial characters. 


\section{BIBLIOGRAPHIE}

JABLOKOFF-KHNZORIAN, S.M., 1963. Nouveau représentants de la tribu des Trechini de l'Arménie soviétique. Zool. Zhurnal, XLII (1):53-61.

JEANNEL, R., 1928. Monographie des Trechinae, 3-me livraison. Les Trechini cavernicoles. $L$ 'A beille, 35: 1-808.

JEANNEL, R., 1947. Coléoptères cavernicoles de l'Anatolie recueillis par M.C.Kosswig. Rev.Fac.Sci.Univ.Istanbul, sér. B, XII (2): 81-88.

JEANNEL, R., 1960. Revision des "Trechini" du Caucase (Coleoptera, Trechidae). Mem.Mus. Nat.d'Hist.Natur.Paris, ser.A, XVII (3): 155-216.

KURNAKOV, V., 1959. Les Trechini de la faune souterraine de l'Abkhazie. Rev.franç. Entomol., XXVI (4): 231-236.

LJOVUSCHKIN, S.I., 1963. On the Trechini fauna from the caves of Western Transcaucasia. Zool. Zhurnal, XLII (3): 451-454.

LJOVUSCHKIN, S.I., 1965. A new species of the troglobiontic caucasian genus Jeannelius (Coleoptera, Trechini) from Anakopian Cave near Novy Afon. Ibid.: XLIV (8): 1262-1265.

LJOVUSCHKIN, S.I., 1970. New forms of Trechini (Coleoptera) from caves of the West Transcaucasia. Ibid.: XLIX (11): 1656-1662. 